\title{
Evaluation of intra-lesional corticosteroids in the treatment of peri-ocular haemangioma of infancy: still an alternative besides propranolol
}

\author{
Sherief R. Janmohamed • Gerard C. Madern • \\ Klaske Nieuwenhuis • Peter C. J. de Laat • \\ Arnold P. Oranje
}

Accepted: 29 November 2011 / Published online: 27 December 2011

(C) The Author(s) 2011. This article is published with open access at Springerlink.com

\begin{abstract}
Purpose Haemangioma of infancy (HOI) is the most frequently occurring benign tumour of infancy. Alarming HOI require treatment. Current therapy is empirically based; corticosteroids are often administered but in recent publications propranolol was reported to be more effective. Peri-ocular HOI are highly sensitive to corticosteroids. Our goal was to evaluate the effectiveness of intra-lesional corticosteroids in the treatment of peri-ocular HOI.

Methods We selected all patients with peri-ocular HOI who had only been treated with intra-lesional
\end{abstract}

S. R. Janmohamed and G. C. Madern contributed equally to this work. Gerard C. Madern, Peter C. J. de Laat and Arnold P. Oranje belong to WEVAR team: workgroup on vascular abnormalities Rotterdam (multidisciplinary patient consultation group).

\section{S. R. Janmohamed · G. C. Madern}

Department of Paediatric Surgery, Erasmus MC-Sophia

Children's Hospital, University Medical Center Rotterdam,

Rotterdam, The Netherlands

S. R. Janmohamed · A. P. Oranje

KinderHaven, Havenziekenhuis, Rotterdam, The Netherlands

\section{S. R. Janmohamed · A. P. Oranje $(\bowtie)$}

Division of Paediatric Dermatology, Department of Paediatrics, Erasmus MC-Sophia Children's Hospital, University Medical Center Rotterdam, Room Sp-1528, P.O. Box 2060,

3000 CB Rotterdam, The Netherlands

e-mail: a.oranje@inter.nl.net

K. Nieuwenhuis - P. C. J. de Laat

Department of Paediatrics, Erasmus MC-Sophia Children's

Hospital, University Medical Center Rotterdam,

Rotterdam, The Netherlands

A. P. Oranje

Department of Dermatology, Maasstadziekenhuis,

Rotterdam, The Netherlands corticosteroids at our hospital from 1993 until 2009. Treatment was standardized according to a prospective protocol.

Results A total of $n=34$ patients were included. There were no complications at all after therapy. A second intralesional injection was necessary in five patients. At followup after 6 and 12 months after injection, 94 and $91 \%$ of the patients, respectively, had regression of the HOI. Astigmatism, Haemangioma Activity Score and global assessments all had improved after therapy.

Conclusions This study shows that intra-lesional therapy with corticosteroids is very safe in the treatment of periocular HOI. It remains a good and safe alternative besides propranolol or when propranolol therapy is not possible (e.g. asthma, PHACE syndrome, and certain cardiac diseases).

Keywords Haemangioma - Intra-lesional corticosteroids · Propranolol · Therapy

\section{Introduction}

Haemangioma of infancy (HOI) [1] is the most common, benign, self-limiting tumour of infancy [2,3] and therefore needs no treatment [4]. It is commonly located on the face [5]. However, HOI may cause severe complications [4], generally because of extensive growth or location, leading to (besides psychological problems) life-threatening or disabling complications [6] (e.g. compression of the airways, heart failure, ulceration and visual problems). Amblyopia or reduced vision in the affected eye, secondary to astigmatism or visual deprivation affects $43-60 \%$ of the children with peri-ocular HOI [1]. Of course, such HOI require treatment. However, the current therapy in HOI is empirically based. Systemic or intra-lesional corticosteroid 

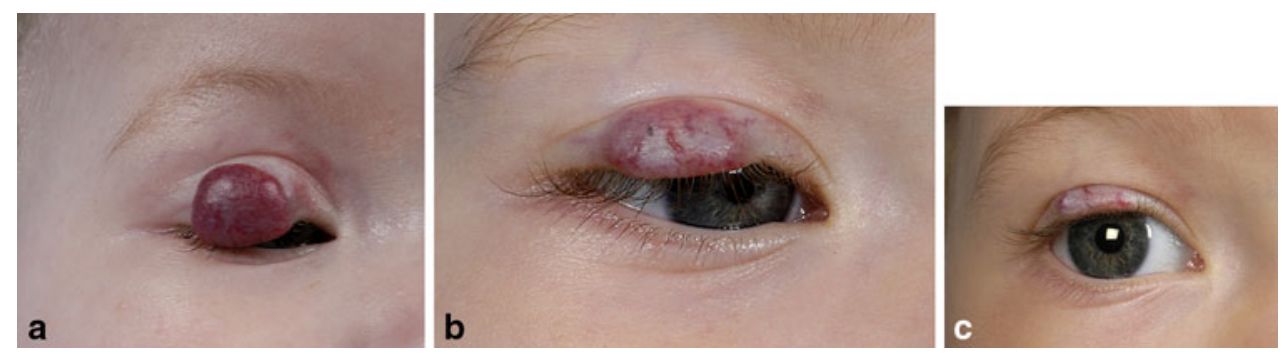

Fig. 1 a A peri-ocular haemangioma of infancy (HOI) in an almost six months old girl at $t=0$. Note the swelling which closes the eye. b Six months after treatment with intra-lesional corticosteroids: the

treatment is often administered [7]. Peri-ocular HOI are particularly sensitive to corticosteroids. In recent publications, propranolol $[8,9]$ was reported to be either more or at least equally effective in treating proliferative HOI. Other alternatives among others are [10] laser therapy, intravenous vincristine, cyclophosphamide and recombinant interferon alfa [11] (high risk for adverse events), local/intra-lesional imiquimod, becaplermin (recombinant platelet-derived growth factor) and bleomycin. Apart from waiting for involution or surgical resection, treatment aims at inducing or accelerating the natural involution process [12]. Although there is extensive literature, objective measures of astigmatism and anisometropia before and after treatment are limited. In this article we evaluate the effectiveness of intra-lesional corticosteroids in the treatment of peri-ocular HOI.

\section{Methods}

Study design and patient selection

In 1993 Oranje, de Laat and Madern started a special outpatient clinic called workgroup on vascular abnormalities Rotterdam (WEVAR) at our hospital, for children with threatening, very large, or fast-growing vascular abnormalities. This outward clinic is multi-disciplinary and includes as a basic team paediatric dermatologist (APO), paediatrician (PCJL) and paediatric surgeon (GCM). Other specialists are consulted if necessary. Treatment (when necessary) is standardized. One of the therapy options is intra-lesional corticosteroids (a mixture of $2 \mathrm{ml}$ kenacort-A $40 \mathrm{mg} / \mathrm{ml}$ and $3 \mathrm{ml}$ celestone $4 \mathrm{mg} / \mathrm{ml}$ ), and these are administered at a dose of $1-5 \mathrm{ml}$ at one or multiple sites, depending on the size of the lesion. Corticosteroids are injected with a 23G $1 \mathrm{in}$. needle. During the procedure, the paediatric surgeon (visually) checks the site of injection. The position of the needle is checked both visually and by palpation. Injection in a great vessel is prevented by checking for blood return in the needle (aspirate). After the
$\mathrm{HOI}$ is in regression and the eye can be opened again. c 12 months after treatment with intra-lesional corticosteroids: the HOI is almost gone

procedure, pressure on the injection site(s) with gauze and using the fingers was performed by the paediatric surgeon until haemostasis had been reached. Standard controls after the procedure consist of checking the site of injection, the eye, general condition, and, by indication, hormonal controls or eye examinations by an ophthalmologist. Figure $1 \mathrm{a}-\mathrm{c}$ show the course of a peri-ocular HOI, treated with intra-lesional corticosteroids.

Patients with alarming (threatening) peri-ocular HOI who needed treatment, e.g. because they were (at risk of) developing amblyopia, were treated according to a WEVAR protocol including ultrasonography of the peri-ocular region and eye examinations prior to therapy. These eye examinations (cycloplegic refractions of both eyes) were also protocolized and performed by a paediatric ophthalmologist. Patients received one drop of a solution containing $2.5 \%$ phenylephrine and $0.5 \%$ tropicamide in each eye. After 15-20 min this was repeated. The examination started 15-20 min after the second drops were administered to the patients. Spherical and cylindrical abnormalities were assessed using skiascopy. All patients were awake during the eye examinations.

\section{Inclusion and exclusion criteria}

All of the patients with alarming peri-ocular HOI (i.e. the eye was at risk because of such threatening HOI) who were treated with intra-lesional corticosteroids from 1993 until 2009 were included in this study. Patients who were treated with both systemic and intra-lesional corticosteroids were excluded, as were patients with HOI at another location than peri-ocular. Patients with intra-orbital HOI are not treated with intra-lesional therapy. Data was obtained by investigating the patients' medical dossiers. Photographs that had been taken were assessed.

\section{Scoring HOI}

The effect of treatment was evaluated by the Haemangioma Activity Score (HAS) [13]. This scoring system focuses on 
Table 1 Demographics and baseline characteristics of $n=34$ patients with peri-ocular HOI treated only with intralesional therapy

${ }^{\text {a }}$ Five patients with a gestational age between 33 and 35 weeks, one patient with a gestational age of $253 / 7$ weeks

\begin{tabular}{ll}
\hline Characteristic & Rx/intra-lesional corticosteroids $(n=34)$ \\
\hline Sex $(\%)$ & Female: 88 \\
& Male: 12 \\
Gestational age (\%) & $>37$ weeks: 82 \\
& Premature: $18^{\mathrm{a}}$ \\
Gemini (\%) & No: 91 \\
& Yes: 9 \\
Median age of development of HOI & 0.4 months $(10$ th percentile $=0.0 ; 90$ th \\
& percentile $=1.75)$ \\
Median number of HOI per patient & $1(10$ th percentile $=1 ; 90$ th percentile $=4)$ \\
Size of HOI (assessed by ultrasonography) & $<3 \mathrm{~cm}: 71$ \\
$(\%)$ & $3-10 \mathrm{~cm}: 26$ \\
& $>10 \mathrm{~cm}: 3$ \\
Median age at start intra-lesional therapy & $3.8 \mathrm{months}(10$ th percentile $=2 ; 90$ th percentile $=9)$ \\
\hline
\end{tabular}

the (disease) proliferative activity of a HOI at a certain time point. Scores at multiple time points of one HOI in one patient can be compared with each other.

\section{Outcome measures}

HAS before and after treatment was calculated at $t=0,6$ and 12 months from the photographs. We searched all patients' medical dossiers for complications. The global assessments at the check-ups on the size of the HOI were collected as were the ophthalmic measurements made by the ophthalmologist.

\section{Statistical methods}

SPSS version 15.0.0 was used for the database and statistical measures. Differences in astigmatism, in matters of refractive cylindrical value, and HAS between the three time points were assessed using Friedman's test.

\section{Results}

In the period 1993-2009 a total of $n=67$ patients with HOI were treated with intra-lesional corticosteroids. After excluding patients with concomitant HOI other than periocular HOI $(n=28)$ and patients who had both systemic and intra-lesional therapy $(n=5)$, a total of $n=34$ patients were included. These patients were treated with only intra-lesional corticosteroids because of an alarming peri-ocular HOI. Their baseline characteristics are shown in Table $1.88 \%$ of patients were females and $82 \%$ full term (gestational age $>37$ weeks). $9 \%$ of patients were twins. The mean age at which the HOI started to develop was 0.63 months (SD 0.67 months). The number of HOI in
Table 2 Outcomes of intralesional corticosteroid therapy in $n=34$ patients with alarming peri-ocular haemangioma of infancy (HOI) at $t=0$ (baseline, just before intra-lesional therapy), 6 and 12 (6 and 12 months after injection)

\footnotetext{
$* p<0.001$

$* * p=0.011$
}

\begin{tabular}{llll}
\hline & $t=0$ & $t=6$ months & $t=12$ months \\
\hline Mean HAS & 3.0 (SD 1.3) & 2.1 (SD 0.9) & 1.6 (SD 0.7)* \\
Refraction: mean astigmatism (diopters) & 2.57 (SD 1.49) & 1.84 (SD 1.01) & 1.44 (SD 1.26)** \\
Occlusion therapy (for amblyopia) (\%) & & & 28 \\
Yes & 46 & 40 & 72 \\
No & 54 & 60 & 91 \\
Therapy effect (evaluation by the doctor) (\%) & & 6 \\
Smaller & 94 & 3 \\
Same & 6 & 0 \\
Worse & 0 & 100 \\
Complications (\%) & & 9 \\
Yes & 0 & 81 \\
No & 100 & \\
Plastic surgery required until 2009? (\%) & & \\
Yes & & \\
No & & & \\
\hline
\end{tabular}




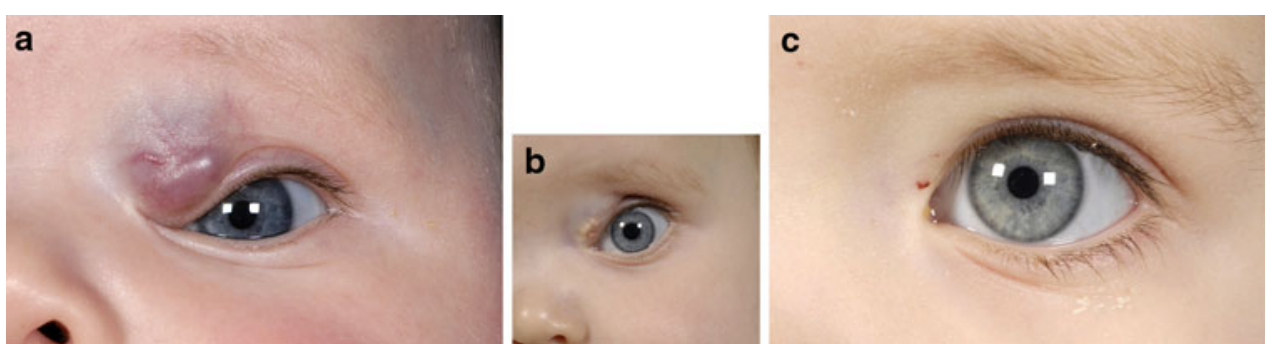

Fig. 2 a A peri-ocular haemangioma of infancy (HOI) in a four months old boy at $t=0$. b Six months after intra-lesional corticosteroids: the HOI is in regression. Note the peri-ocular

each patient ranged from 1 to 5 but most patients had 1 HOI (median number $=1$, 10th percentile $=1$, 90th percentile $=4)$ and most $\mathrm{HOI}$ were small $(71 \%<3 \mathrm{~cm}$, $3 \%>10 \mathrm{~cm}$, greatest diameter taken on ultrasonography). Finally, the median age of first intra-lesional therapy was 3.8 months (10th percentile $=2$, 90th percentile $=9$ ).

Of these $n=34$ patients, 5 patients were given a second intra-lesional corticosteroid injection. Table 2 shows the outcomes of the therapy at 6 and 12 months after the first injection. HAS declined from 3 (SD 1.3) at baseline to 2.1 (SD 0.9) after 6 months and 1.6 (SD 0.7) after 12 months after the first injection $(p=0.000)$. Global scores by the doctor strengthen the (reduction in) HAS: after 6 months 94\% was scored as 'smaller', after 12 months this was $91 \%$ (6\% was the same and $3 \%$ worse). Astigmatism shows a similar trend as the HAS: mean cylindrical diopters dropped from 2.57 (SD 1.49) at baseline to 1.84 (SD 1.01) after 6 months and 1.44 (SD 1.26) after 12 months ( $p=0.011$ ). At baseline, $46 \%$ of the patients had occlusion therapy, after 6 months $40 \%$ and after 12 months only $28 \%$ of all patients still had occlusion therapy. Until now, $9 \%$ of patients had plastic surgery. Most importantly we did not see any (lasting) complications. In one patient we did see peri-ocular calcification, but this appeared to be reversible.

\section{Discussion}

This study showed that intra-lesional therapy with corticosteroids is a very safe alternative in the treatment of (peri-ocular) HOI as no complications were observed by us. HAS, eye examinations, and clinical appearance all improved after intra-lesional therapy. Since HOI is a selflimiting tumour, the expectation is that HAS and clinical appearance would eventually improve, but we were able to demonstrate that when an eye is at risk of visual impairment, intra-lesional therapy is highly effective: all eye examinations (especially cylindrical abnormalities) improved after treatment.

In $9 \%$ of our cases, plastic surgery was indicated in a later stage. However, this did not mean that intra-lesional calcification. c Two years after intra-lesional corticosteroids: the peri-ocular calcification appeared to be reversible

therapy was not effective. The HOI did regress faster. We think that without the intra-lesional therapy or with another therapy, a scar would have eventually developed that required plastic surgery. It is possible that this percentage may have been higher because some patients could have gone to another hospital or may require plastic surgery in the future.

Our results of the treatment with intra-lesional corticosteroids for peri-ocular HOI are excellent with a success rate of $85 \%$ after one injection and $100 \%$ after two injections, without any adverse events. These percentages are somewhat higher than those reported in the literature. 50\% of HOI respond after therapy with corticosteroids, periocular HOI are more sensitive, with rates up to $64 \%$ [10, $14,15]$. These differences are probably attributed to other measures of effect and success. Often, the amount of corticosteroids that were administered in those cases is also difficult to establish. We treated all patients in a standardized manner.

The possible side effects are as follows: injection pressure during intra-lesional injection usually exceeds systemic arterial pressure and poses a risk of corticosteroid particle embolization into the ocular circulation due to retrograde arterial flow. Serious ocular complications include ophthalmic artery occlusion, retinal embolization and central retinal artery occlusion. Others are eyelid hypopigmentation, linear subcutaneous fat atrophy, sclerodermiform linear atrophy, eyelid necrosis and peri-ocular calcification. Rare systemic side effects include cushingoid features, growth deceleration and adrenal suppression [10, 15]. We did not encounter any of these side effects. Periocular calcification was seen in one patient but appeared to be reversible (see Fig. 2a-c). The most important thing is to aspirate for blood after injection and inject with care. For example, if the HOI is located in the eyeball, intra-lesional therapy is dangerous because the HOI expands while corticosteroids are administered leading to compromised perfusion and visual loss. An extension of the HOI into the orbital cavity can also be associated with complications, and therefore we do not treat such patients with intra-lesional therapy. 
Table 3 Advantages and disadvantages of intra-lesional corticosteroid treatment and propranolol treatment of peri-ocular haemangioma of infancy (HOI)

\begin{tabular}{|c|c|c|}
\hline & Intra-lesional corticosteroids & Propranolol \\
\hline \multirow[t]{5}{*}{ Advantages } & $\begin{array}{l}\text { Often just one injection, instead } \\
\text { of long-term administration of medicine }\end{array}$ & $\begin{array}{l}\text { Seems to work at least as good as intra-lesional } \\
\text { corticosteroids } \\
\text { without the side effects of intra-lesional therapy }\end{array}$ \\
\hline & Local, once & \\
\hline & Good response on peri-ocular HOI & \\
\hline & $\begin{array}{l}\text { A lot of experience with this therapy: not many } \\
\text { side effects }\end{array}$ & \\
\hline & $\begin{array}{l}\text { Can be given when propranolol fails, } \\
\text { or in PHACE syndrome }\end{array}$ & \\
\hline \multirow[t]{5}{*}{ Disadvantages } & Anaesthesia is recommended to minimize side effects & Systemic \\
\hline & Other HOI than peri-ocular have a success rate of only $50 \%$ & $\begin{array}{l}\text { Side effects have not yet been properly investigated } \\
\text { at this age (watch out: do not give in patients with PHACE. } \\
\text { Several published case reports with hypoglycaemia). } \\
\text { Blue coloured hands and feet }\end{array}$ \\
\hline & 1 day hospitalization, limited ultrasound evaluation & $\begin{array}{l}1 \text { day hospitalization, expensive multiple ultrasound } \\
\text { pre-treatment evaluation }\end{array}$ \\
\hline & $\begin{array}{l}\text { Sometimes the therapy has to be repeated } \\
\text { later on (with another narcosis) }\end{array}$ & $\begin{array}{l}\text { Doses and duration of therapy is still unknown. } \\
\text { Additional investigations are necessary }\end{array}$ \\
\hline & $\begin{array}{l}\text { When administered by non-experienced physicians: } \\
\text { retinal occlusion/optic nerve compression is a rare } \\
\text { complication }\end{array}$ & $\begin{array}{l}\text { Contra-indicated in vascular malformations of internal } \\
\text { organs and PHACE syndrome [16] }\end{array}$ \\
\hline
\end{tabular}

Furthermore, female to male ratio is slightly high in our study, but agrees with the reported findings that HOI is more common in girls [12]. We also observed that most patients had a small $(<3 \mathrm{~cm})$ HOI.

In spite of these good results, intra-lesional therapy of HOI with corticosteroids is undergoing a transformation. Today there is an increasing interest on propranolol in the treatment of HOI with promising results and until now few reported side effects. We think that it has almost become the first choice of treatment. The results of this study showed that intra-lesional injection with corticosteroids is still a safe alternative for treating (peri-ocular) HOI when propranolol is not effective or when propranolol is (relatively) contra-indicated (e.g. asthma, certain cardiac diseases, multiple medication causing extra burden and PHACE syndrome [16]). The recent study reported by Greenberger et al. [17] elucidated the effect of corticosteroids on angiogenesis and vasculogenesis. Certain advantages and disadvantages of both propranolol and intralesional therapy are summarized in Table 3 . We conclude that intra-lesional therapy with corticosteroids still has a valuable place in the treatment of HOI.

Conflicts of interest The authors declare that they have no conflict of interest.

Open Access This article is distributed under the terms of the Creative Commons Attribution Noncommercial License which permits any noncommercial use, distribution, and reproduction in any medium, provided the original author(s) and source are credited.

\section{References}

1. Bruckner AL, Frieden IJ (2003) Hemangiomas of infancy. J Am Acad Dermatol 48(4):477-493 (quiz 494-476)

2. Boye E, Olsen BR (2009) Signaling mechanisms in infantile hemangioma. Curr Opin Hematol 16(3):202-208

3. Chang EI, Chang EI, Thangarajah H, Hamou C, Gurtner GC (2007) Hypoxia, hormones, and endothelial progenitor cells in hemangioma. Lymphat Res Biol 5(4):237-243

4. Bruckner AL, Frieden IJ (2006) Infantile hemangiomas. J Am Acad Dermatol 55(4):671-682

5. Lo K, Mihm M, Fay A (2009) Current theories on the pathogenesis of infantile hemangioma. Semin Ophthalmol 24(3): 172-177

6. Sun ZY, Yi CG, Zhao H, Yin GQ, Gao M, Liu YB, Qin JD, Wang SF, Guo SZ (2008) Infantile hemangioma is originated from placental trophoblast, fact or fiction? Med Hypotheses 71(3): 444-448

7. Kushner BJ (1982) Intralesional corticosteroid injection for infantile adnexal hemangioma. Am J Ophthalmol 93(4):496-506

8. Leaute-Labreze C, Dumas de la Roque E, Hubiche T, Boralevi F, Thambo JB, Taieb A (2008) Propranolol for severe hemangiomas of infancy. N Engl J Med 358(24):2649-2651

9. Sans V, de la Roque ED, Berge J, Grenier N, Boralevi F, Mazereeuw-Hautier J, Lipsker D, Dupuis E, Ezzedine K, Vergnes $\mathrm{P}$, Taïeb A, Léauté-Labrèze C (2009) Propranolol for severe infantile hemangiomas: follow-up report. Pediatrics 124(3): e423-e431

10. Weiss AH, Kelly JP (2008) Reappraisal of astigmatism induced by periocular capillary hemangioma and treatment with intralesional corticosteroid injection. Ophthalmology 115(2):390-397 e391

11. Lawley LP, Siegfried E, Todd JL (2009) Propranolol treatment for hemangioma of infancy: risks and recommendations. Pediatr Dermatol 26(5):610-614 
12. Boye E, Jinnin M, Olsen BR (2009) Infantile hemangioma: challenges, new insights, and therapeutic promise. J Craniofac Surg 20(Suppl 1):678-684

13. Janmohamed SR, de Waard-van der Spek FB, Madern GC, de Laat PC, Hop WC, Oranje AP (2011) Scoring the proliferative activity of haemangioma of infancy: the Haemangioma Activity Score (HAS). Clin Exp Dermatol 36(7):715-723

14. Verity DH, Rose GE, Restori M (2008) The effect of intralesional steroid injections on the volume and blood flow in periocular capillary haemangiomas. Orbit 27(1):41-47
15. Nguyen J, Fay A (2009) Pharmacologic therapy for periocular infantile hemangiomas: a review of the literature. Semin Ophthalmol 24(3):178-184

16. Sidbury R (2010) Update on vascular tumors of infancy. Curr Opin Pediatr 22(4):432-437

17. Greenberger S, Boscolo E, Adini I, Mulliken JB, Bischoff J (2010) Corticosteroid suppression of VEGF-A in infantile hemangioma-derived stem cells. N Engl J Med 362(11):1005-1013 